\title{
Learning by Selection: Visual Search and Object Perception in Young Infants
}

\author{
Dima Amso \\ Sackler Institute for Developmental Psychobiology, \\ Weill Medical College of Cornell University
}

\author{
Scott P. Johnson \\ New York University
}

\begin{abstract}
The authors examined how visual selection mechanisms may relate to developing cognitive functions in infancy. Twenty-two 3-month-old infants were tested in 2 tasks on the same day: perceptual completion and visual search. In the perceptual completion task, infants were habituated to a partly occluded moving rod and subsequently presented with unoccluded broken and complete rod test stimuli. In the visual search task, infants viewed displays in which single targets of varying levels of salience were cast among homogeneous static vertical distractors. Infants whose posthabituation preference indicated unity perception in the completion task provided evidence of a functional visual selective attention mechanism in the search task. The authors discuss the implications of the efficiency of attentional mechanisms for information processing and learning.
\end{abstract}

Keywords: visual attention, infancy, object perception, oculomotor control

The first step in perception and learning is the acquisition of information. Infants gather information from the environment using a variety of methods, including manual exploration, crawling, reaching, sucking, and listening. Shifts of eye gaze, in particular, provide a rich source of information about the visual world, and infants engage in active visual exploration from birth (Slater, 1995). A great deal of research has focused on the development of oculomotor control and visual selection, but little is known about their contributions to learning and other kinds of cognitive development in infancy. In the present article, we examine how visual selective attention may relate to emerging cognitive skills in infancy.

Oculomotor control develops rapidly in infancy. Initially, many shifts of visual attention are guided reflexively, or exogenously, largely by stimulus salience. Voluntary, or endogenous, control over visual selection improves across the first several postnatal months (M. H. Johnson, 1990). Selective attention has been described as one or more mechanisms that determine what information from the external environment enters a system for subsequent processing (Craik, Govoni, Naveh-Benjamin, \& Anderson, 1996; Treisman, 1992). Visual selective attention, for example, supports the selection of certain stimuli for processing while potentially interfering stimuli are ignored, a candidate skill in controlled visual exploration (Amso \& Johnson, 2005).

Dina Amso, Sackler Institute for Developmental Psychobiology, Weill Medical College of Cornell University; Scott P. Johnson, Department of Psychology, New York University.

This research was supported by National Science Foundation Grant BCS-0418103 and National Institutes of Health Grant R01-HD40432. We gratefully acknowledge the efforts of the infants and parents who paticipated in the sudies. We also thank Myque Harris and Kristin Bellanca for invaluable help recruiting the infant participants.

Correspondence concerning this article should be addressed to Dima Amso, Sackler Institute for Developmental Psychobiology, Weill Medical College of Cornell University, 1300 York Avenue, Box 140, New York, NY 10028. E-mail: dia2004@med.cornell.edu
A principal idea in the study of information-gathering behaviors in infancy has been that the acquisition of new motor skills opens up avenues to novel exploratory situations, which then influence cognitive development (Bushnell \& Boudreau, 1993; Gibson, 1988). This idea has been supported by a growing body of work showing that infants' perceptual, cognitive, and action-based abilities seem all to be related in meaningful ways (e.g., Adolph, 1997, 2000; Gibson \& Pick, 2000). For example, research investigating the influence of manual exploration on cognitive and perceptual judgments has shown that infants who engage in more active exploration are better able to segregate an object into its component parts than infants who engage in less active exploration strategies (Needham, 2000; Needham, Barrett, \& Peterman, 2002). Similarly, a recent study that investigated object perception in young infants (S. P. Johnson, Slemmer, \& Amso, 2004) revealed a relation between visual exploration and the ability to bridge the gaps imposed by occlusion, a phenomenon known as perceptual completion.

Perceptual completion develops across the first several months after birth. Neonates have been shown consistently to respond to partly occluded object displays, such as those depicted in Figure 1, only in terms of what is directly visible, failing to perceive the unity of the top and bottom portions of a center-occluded object (Slater, Johnson, Brown, \& Badenoch, 1996; Slater et al., 1990). That is, infants begin postnatal life with the ability to achieve figure-ground segregation, and they perceive visible surfaces as distinct by virtue of differences in color, luminance, texture, and other visual cues. Neonates, however, do not appear to perceive occlusion, so visible surfaces may constitute sensorial images with no depth, solidity, or volume (S. P. Johnson, 2000; Piaget, 1937/ 1954). By 2 months, infants perceive unity under limited conditions, and by 4 months, perceptual completion is more robust (S. P. Johnson, 2004).

S. P. Johnson et al. (2004) examined perceptual completion in a group of 3-month-old infants. The infants were habituated to a 


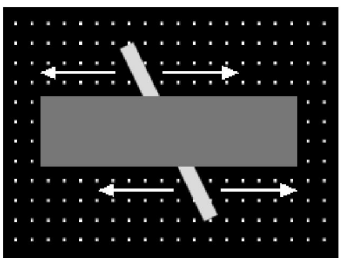

A

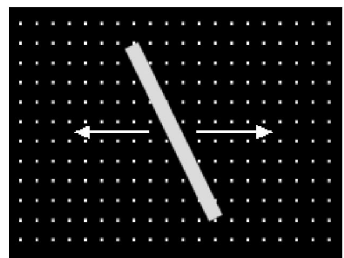

$\mathrm{B}$

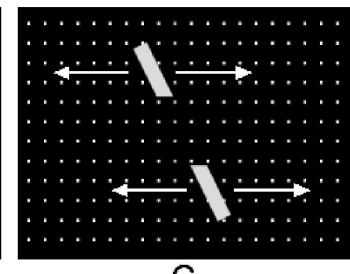

C

Figure 1. Schematic depictions of displays presented to infants in Experiment 1. Figure 1A: habituation display, consisting of a partly occluded rod. Figure 1B: complete rod test stimulus. Figure 1C: broken rod test stimulus.

partly occluded rod display, followed by two test displays (Figure 1). The complete rod test display was designed to match a percept of unity, and the broken rod test display was designed to match a percept of disjoint rod surfaces. Longer looking toward one of the two test displays was interpreted as a posthabituation novelty preference (Bornstein, 1985; Sokolov, 1963). A preference for the broken rod, therefore, was taken to suggest perception of unity of the rod parts during habituation. In addition to this measure of unity perception, infants' oculomotor scanning patterns were recorded with a corneal reflection eye tracker as the infants viewed the rod-and-box habituation display. Those infants whose posthabituation looking times implied unity percepts (i.e., infants who preferred the broken rod, termed perceivers) fixated the rod more frequently and scanned more often across the rod's path as it translated back and forth, relative to the infants whose test display preference did not imply perception of the partly occluded rod as unified (termed nonperceivers).

S. P. Johnson et al. (2004) speculated that the scanning patterns exhibited by the perceivers served to maximize information uptake about the features of the habituation stimulus that were relevant for perceptual completion, features such as the alignment of the visible rod parts and their motions. Identification of and attention toward these features might have supported unity perception, perhaps more so than would be possible via a focus on display features that were less relevant to unity (e.g., the occluding box or the background). More efficient scanning in this task (i.e., visually orienting to relevant parts of the display and disregarding others) may be a manifestation of some underlying, independent ability. A logical candidate is the change in oculomotor control from reflexive to controlled visual orienting in this age range. In the present study, we use an independent visual search task to investigate group differences between infants who provide evidence of unity perception and efficient visual exploratory behavior during the habituation phase of the object unity paradigm and those who do not. We predict group differences in visual search performance between perceivers and nonperceivers if developments in visual attention contribute to perception of object unity.

Investigations of visual selective attention have often used search tasks in which a unique target element is cast in an array of homogeneous distractors (Neisser, 1967; Treisman, 1988; Treisman \& Gelade, 1980). Treisman and Gormican (1988), for example, presented to adults an oblique target among vertically oriented distractors. The observers detected the oblique target consistently and rapidly, and reaction times were unaffected by the number of distractors in the display, a phenomenon known as pop-out. The pop-out effect has been attributed to a processing mechanism that identifies the location of a salient item during the early, preatten- tive stages of visual processing (Treisman, 1988; Treisman \& Gelade, 1980). In Treisman's (1988) original account, this “parallel" processing stage is followed by a limited-capacity, slower, attention-directed "serial" stage that requires the deployment of attention from one item to the next until the target is detected. This parallel-serial dichotomy is not accepted universally. Search performance instead may be better characterized as a continuum of target-distractor similarity. Regardless, there are performance differences between searches involving a target that is extremely salient relative to the background and searches in which an increase in target-background similarity heightens the difficulty of target detection and requires efficient selective attention (Duncan \& Humphreys, 1989; Wolfe, 1998). For example, the latency to select a target increases as the target becomes increasingly more similar to the background or if additional distractor elements are added to the background.

Developmental research using novelty preference paradigms (Colombo, Ryther, Frick, \& Gifford, 1995; Quinn \& Bhatt, 1998) and the mobile conjugate reinforcement method (Adler, Gerhardstein, \& Rovee-Collier, 1998; Adler, Inslicht, Rovee-Collier, \& Gerhardstein, 1998; Bhatt, Rovee-Collier, \& Weiner, 1994; Rovee-Collier, Bhatt, \& Chazin, 1996; Rovee-Collier, Hankins, \& Bhatt, 1992) has provided evidence of sensitivity to pop-out as early as 3 months of age. In the natural environment, of course, many salient elements are visible simultaneously. Attending to a particular location in a display, therefore, must necessarily involve inhibiting other potentially salient locations. In the absence of some endogenous controlling mechanism, responses to all salient information in a visual scene may be maladaptive, perhaps impeding learning of any particular element as attention is directed from one item to the next. A key characteristic of an efficient attentional mechanism underlying learning must involve more than a reflexive response to a unique element. It must involve, when necessary, the capacity to resolve the attentional competition between unique elements.

Experiments that explore sensitivity to competition between parts of a visual scene in infants have made use of visual search tasks similar to those used with adult participants. Dannemiller $(1998,2000,2002)$ described a paradigm in which a moving target was embedded in an array of static red and green distractors that were evenly distributed on the left and right hemifields of a display. Young infants (2- to 5-month-olds) were observed for evidence of target detection. The likelihood that infants fixated the target was influenced by the spatial distribution of the distractors: Orienting was weakened when relatively high-salience bars were placed contralateral to the moving target. Salience was determined by the contrast of distractors (Ross \& Dannemiller, 1999); high- 
contrast stimuli are more likely to draw attention (Zenger, Braun, $\&$ Koch, 2000). Thus, attention tended to be divided more evenly between hemifields when salient features, such as static highcontrast bars, competed with the moving target. Sensitivity to competition (i.e., reduced orienting to the moving probe in the face of competition), therefore, was shown to improve across the first 5 months after birth (Dannemiller, 2000), consistent with the possibility that developing visual selective attention mechanisms were responsible for the effect.

Several lines of evidence suggest that there may be a link between developments in emerging visual selective attention and perceptual completion. First, infants who indicated perception of the unity of the rod parts attended to the relevant parts of the display (S. P. Johnson et al., 2004), which implies a functional mechanism that resolves attentional competition between regions in a display (cf. Dannemiller, 2000). Second, 2-month-olds provided evidence of perception of unity only when the occluder was relatively narrow or when gaps were placed strategically in the box such that more of the translating rod was visible (S. P. Johnson, 2004; S. P. Johnson \& Aslin, 1995). It is possible, therefore, that experimental manipulations that increase the salience of the most relevant environmental feature (in this case, the moving rod) obviate the need for a functional selective attention mechanism to accomplish the task. Third, developments in perceptual completion between 2 and 4 months occur in parallel with changes documented in the development of attentional skills in infancy. The frequency with which infants switch attention undergoes substantial development early in postnatal life (Bronson, 1994; M. H. Johnson \& Tucker, 1996). The period between 2 and 4 months appears to be particularly important in the transition to adultlike selective attention, as indexed by how well infants disengage one point of regard to engage another (Atkinson, Hood, Wattam-Bell, \& Braddick, 1992). Finally, other kinds of exploratory movement in infancy, such as locomotor control, show a parallel developmental pattern, from early, spontaneous wiggles or thrashes toward more attentive, controlled movements that are presumably targeted at extracting information from the environment (Adolph, Eppler, Marin, Weise, \& Clearfield, 2000).

We tested 3-month-old infants in a visual search paradigm with two conditions aimed at engaging both visual selective attention and simple orienting to salient stimuli. The paradigm was adapted to accommodate the question of interest: How do infants direct visual attention as display elements vary along the salience dimension? Search task difficulty is largely affected by the similarity of target and distractor items (e.g., Duncan \& Humphreys, 1989), with increased similarity resulting in greater competition for attentional resources. In our task, the competition condition consisted of a field of static, homogeneous vertical bars with a single target bar, tilted from the vertical, at one of three possible orientations. The control condition was identical to the competition condition, except that the target was vertically oriented and translated laterally at one of three possible speeds. We reasoned that performance in the competition condition would require visual selective attention, as indexed by increased latency for target selection, whereas the control condition would require simple reflexive orienting. A developmental shift in oculomotor control from reflexive orienting to controlled visual attention has been reported in the 3-4-month age range (M. H. Johnson, 1990). As noted, as target-distractor similarity increases, so does latency to select the target. Infants who use selective attention to visually explore should be affected by this increase in target-distractor similarity in the competition condition by showing an increase in response latency, relative to those who rely largely on reflexive orienting.

We also tested the same infants, on the same day, in a replication of the S. P. Johnson et al. (2004) experiment: Infants were habituated to a rod-and-box display, followed by broken and complete rod test displays, as eye movements were recorded with an eye tracker. If perceptual completion is related to controlled visual exploration, then infants who provide evidence of perceiving the unity of rod parts should show efficient scanning patterns during habituation (S. P. Johnson et al., 2004) as well as visual search behavior indicative of visual selective attention.

\section{General Method}

\section{Participants}

Twenty-two 3-month-old infants (age, $M=96.3$ days, $S D=7.0$ days; 13 girls) composed the final sample. Thirty infants were observed but excluded from the sample because of the completion of one task but not the other (10 infants), general fussiness (5 infants) or sleepiness (2 infants), program error during data collection ( 9 infants), experimenter error (2 infants), or uninterpretable eye movement data resulting from poor calibration of the point of gaze (POG; 3 infants). All infants were born at term with no known developmental difficulties. Infants were recruited from a public database of new parents, and parents were contacted by letter and telephone. Information on family race/ethnicity, size, socioeconomic status, and education was not available for this sample. Parents were offered compensation for travel expenses and provided a token thank-you gift (a toy or T-shirt with the lab logo for the infant).

\section{Eye Tracking Procedure}

Infants were tested individually, seated $120 \mathrm{~cm}$ from a $76-\mathrm{cm}$ monitor used to present the stimuli. Eye movements were recorded with a remoteoptics corneal reflection eye tracker (Applied Science Laboratories Model 504, Bedford, MA). Each infant's POG was calibrated with an attentionattracting stimulus that contracted and expanded in synchrony with a rhythmic sound at the top left and bottom right corners of an imaginary rectangle that contained the possible stimulus locations. Infants then viewed the calibration stimulus at several random locations on the screen. If the POG was not within $0.5^{\circ}$ of the center of the attention-getter at all locations (minimum of six), the calibration procedure was repeated. Once the calibration criterion had been reached, the experiment began. Data (the POG superimposed on the stimuli) were recorded onto both digital videotape and the computer's hard drive and were coded offline.

\section{Experiment 1: Perceptual Completion}

\section{Method}

Apparatus and stimuli. The habituation display (see Figure 1) consisted of a $36.5 \times 10.4 \mathrm{~cm}$ blue box $\left(17.3^{\circ} \times 5.0^{\circ}\right.$ visual angle at the infant's $120-\mathrm{cm}$ viewing distance) and a $2.3 \times 26.2 \mathrm{~cm}$ green rod $\left(1.1^{\circ} \times\right.$ $\left.12.5^{\circ}\right)$ that translated laterally through $17.7 \mathrm{~cm}\left(8.4^{\circ}\right)$. Each cycle of motion lasted $5 \mathrm{~s}$ (i.e., $2.5 \mathrm{~s}$ either left or right). The rod thus moved at a rate of $7.1 \mathrm{~cm} / \mathrm{s}(3.4 \%)$. The rod moved back and forth continuously as long as the stimulus was shown. The two test displays (see Figure 1) were identical to the habituation display, except that the complete rod had no box but instead the visible rod parts were connected, and the broken rod had a gap between the rod parts in which the background texture was visible. Objects were presented against a black background with a $12 \times 20$ grid of white dots $\left(43.8 \times 30.2 \mathrm{~cm}, 20.7^{\circ} \times 14.3^{\circ}\right)$. 
Procedure. Two experimenters, the habituation experimenter and the eye tracker experimenter, worked in concert to collect looking time data and record eye movements, respectively. The habituation experimenter was blind to test stimulus order, did not know when the displays changed from habituation to test, and had no access to the gaze coordinates calculated by the eye tracker. The eye tracker experimenter had full information about the stimulus seen by the infant at any given time but had no influence over the stimulus or over the habituation experimenter's judgments of looking times. Each trial commenced with presentation of the attention-getter. The habituation experimenter ended the attention-getter and began the stimulus for each trial when it was judged that the infant looked at the display. A trial ended when the infant looked away for $2 \mathrm{~s}$ or when $60 \mathrm{~s}$ had elapsed; the stimulus was then replaced by the attention-getter to begin the next trial. The habituation stimulus was presented until looking times declined across 4 continuous trials that summed to less than half the total during the first 4 trials. The minimum number of habituation trials was 5 , and the maximum was 12. Infants viewed the test displays three times each in alternation. These methods are identical to those described in the S. P. Johnson et al. (2004) experiment. Experiment order was randomized: Ten infants participated in the perceptual completion experiment first, followed by the visual search experiment, and 12 participated in the reverse order. Half the infants in each experiment order viewed the broken rod first after habituation, and half viewed the complete rod first.

\section{Results}

A reliable preference for the broken rod test display was taken as evidence for perceptual completion in the habituation display. This preference was computed as the ratio of looking times toward the broken rod as a function of total looking times to both test displays. Across the sample, there was no consistent preference for either test display (preference for broken, $M=53.6 \%, S D=$ $14.4 \%$ ), which replicates the outcome of the S. P. Johnson et al. (2004) experiment. Preference data from the group of 22 infants were divided on the mean (which was the same as the median in this case) for subsequent analysis. Infants whose posthabituation preference exceeded the mean (whom, as in the S. P. Johnson et al. study, we termed perceivers; 7 girls and 4 boys; age, $M=95.1$ days, $S D=6.7$ ) preferred the broken rod (preference for broken, $M=65.2 \%, S D=8.5 \%), t(10)=3.03, p<.05$. The perceivers dishabituated to the first broken rod test display, $t(10)=3.47, p<$ .001 , showing a significant increase in looking time relative to the last habituation trial, but did not recover interest to the first complete rod display, $t(10)=1.97$, ns. Infants whose preference was less than the mean (whom we termed nonperceivers; 6 girls and 5 boys; age, $M=97.6$ days, $S D=7.4$ ) looked longer at the complete rod overall (preference for broken, $M=42.0 \%, S D=$ $8.5 \%), t(10)=2.70, p<.05$, but provided no evidence of dishabituation to either test display, $t \mathrm{~s}(10)<0.5$, ns. This suggests that this group might have expressed perceptual uncertainty during test rather than unequivocally perceiving disjoint surfaces. The difference in mean preference between the two groups was significant, $t(20)=6.37, p<.0001$ (see Figure 2). There were no reliable differences between groups in terms of sex (i.e., distribution of girls and boys, as indexed by chi-square), age, total time to habituate $(M=162.6 \mathrm{~s}, S D=99.8 \mathrm{~s}$, across the sample), total looking time during test $(M=68.1 \mathrm{~s}, S D=65.2 \mathrm{~s})$, or the amount of eye tracking data from each infant as a proportion of total looking time judged by the habituation experimenter $(M=32.4 \%$, $S D=14.2 \%$; all $t \mathrm{~s}<1.85, n s$ ). A 2 (group: perceivers vs. nonperceivers) $\times 2$ (task order: perceptual completion vs. visual

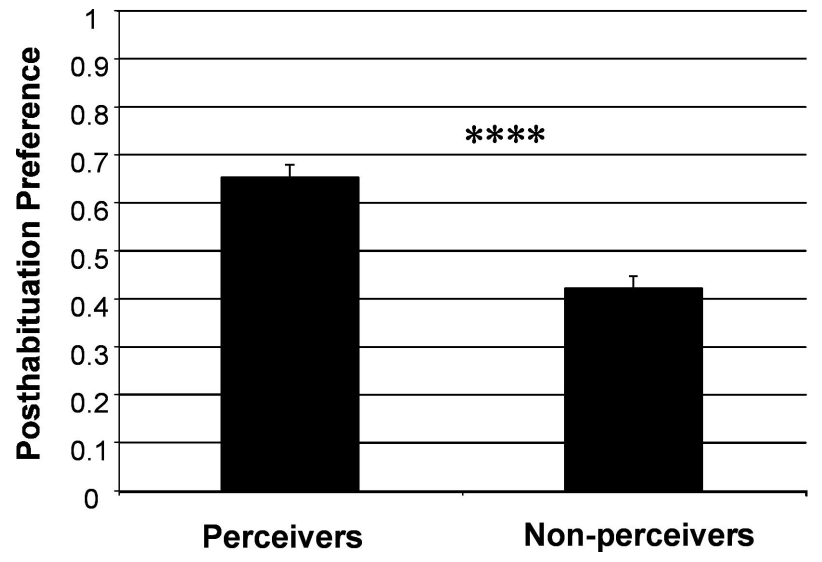

Figure 2. Mean posthabituation preferences for the broken rod test display in Experiment 1. Error bars indicate standard error of measurement. $* * * * p<.0001$.

search) $\times 2$ (test display order: broken vs. complete first $) \times 2$ (display: broken vs. complete) $\times 3$ (trial block) mixed analysis of variance (ANOVA) yielded no reliable main effects or interactions except a Group $\times$ Test display interaction, $F(1,14)=10.98, p<$ .01. This interaction reflects the difference in performance between perceivers and nonperceivers described previously.

Eye tracking data were examined for scanning differences between groups. (Two infants produced a disproportionately small amount of eye movement data during habituation and were excluded from the following analyses). Eight areas of interest (AOIs) were defined with respect to the boundaries of the two visible rod parts in the habituation display, the left and right halves of the occluding box, and the four quadrants of the background excluding the rod parts and box (see Figure 3). We determined each infant's scanning pattern by summing the number of saccades that began and ended in each of the eight AOIs. Following S. P. Johnson et al. (2004), we reasoned that there would be individual differences in the extent to which infants would tailor scanning characteristics to the unity task and that scan patterns indicative of efficient information gathering via visual exploration would involve greater visual attention to the rod parts and their motion above and below the occluder. Especially pertinent to the unity task is the extent to which infants scan in the vicinity of the rod. We defined rod scans as the proportion of saccades that began and ended within the boundaries encompassing one or both visible rod parts or that took the POG across the rod parts' range of motion (e.g., a saccade from the lower left to the lower right quadrant or vice versa). We reasoned, furthermore, that, in contrast to the predictions about rod scans, there would be no systematic differences between groups in scanning patterns that were presumably unrelated to unity perception, such as vertical scans, defined as the proportion of saccades that brought the POG from a top to a lower quadrant (not across a rod AOI or across the rod's path of motion).

Consistent with our predictions, perceivers produced more rod scans than nonperceivers, $t(18)=2.11, p<.05$ (see Figure 4 ), but there was no reliable difference in vertical scans, $t(18)=0.37, n s$. The correlation between individual infants' rod scans and posthabituation preference was marginally significant, $r(18)=.44, p=$ .053 , indicating that although visual exploration (orienting to the 


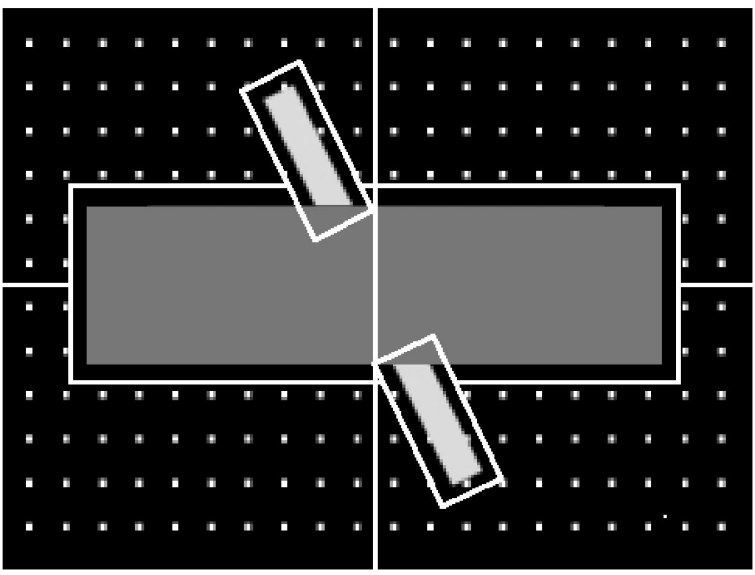

Figure 3. The rod-and-box display presented to infants during habituation in Experiment 1. The grey outlines represent the areas of interest (AOIs) defined for eye movement data analyses and were not visible to infants. The AOIs containing the visible rod parts "moved" with the rod for purposes of analysis.

relevant regions of the display during habituation) was related to unity perception, it was neither necessary nor sufficient, an issue we revisit in the General Discussion. Other measures were not reliably different across groups, such as proportion of eye tracking data collected, local versus global scan patterns (i.e., the extent to which saccades were localized within AOIs; $M=50.1 \%, S D=$ $10.7 \%$, across the sample were local scans), and saccades per second as a metric of active scanning $(M=4.57, S D=0.52$, across the sample; $t \mathrm{~s}<1.4, n s$ ).

\section{Discussion}

The principal difference in performance between perceivers and nonperceivers (i.e., infants who provided evidence of perceptual completion and those who did not, respectively) lay in the extent to which the groups attended to informative regions of the display (the moving rod parts). Perceivers did not produce more visual exploratory activity overall but showed rather more targeted or controlled exploration. One possible explanation for the differences between groups appeals to preexisting differences in perceptual completion skills: Like older infants and adults, the perceivers might have entered the task with the ability to represent partly hidden objects as continuous across occlusion, whereas nonperceivers, like younger infants, processed only the visible surface elements in the display, failing to achieve perceptual completion. According to this account, perceivers tailored scanning patterns to the task, but nonperceivers had little reason to attend preferentially to the rod parts to discern their unity.

Alternatively or in addition, the posthabituation looking times and scanning data in this experiment and in the S. P. Johnson et al. (2004) study may be interpreted as suggesting that differences in visual selective attention between the two groups supported distinct strategies of information acquisition during habituation. According to this account, differences in the developmental state of visual selection mechanisms affect what information is acquired. Spatial arrangement (i.e., alignment across the occluder) and motion of the rod parts are known to be informative with respect to perceptual completion in infancy (S. P. Johnson, 2004; Kellman \& Spelke, 1983). The moving rod parts are also highly salient. All infants would be expected to attend to the rod parts if they were the only salient display elements, but other display elements may compete for visual attention as well. The occluder in the habituation display, for example, was bright blue and was substantially larger than the rod (see Figure 1). The background, composed of white, textured display elements arranged in symmetrical rows, extended over more space than did the rod parts. These competing display elements may pose a challenge for a developing visual system that has difficulty inhibiting reflexive eye movements to salient stimuli. A mechanism that can resolve the competition between display elements to track the synchronous common motion of the rod parts would likely provide an important contribution to perceptual completion. Perceivers, therefore, might have been more capable of targeted oculomotor control and thereby attended more to the informative regions of the stimulus. In contrast, nonperceivers might have been more distracted by other salient elements in the display.

S. P. Johnson (1997, 2000) proposed a threshold model positing that object perception depends on the sufficiency of visual information as well as the efficiency of perceptual or cognitive skills. It is possible that the nonperceivers, with relatively inefficient oculomotor mechanisms for targeted visual exploration of a complex visual display, were somewhere between perceiving the occlusion stimulus as fragmented and perceiving it as unified. They might have looked longer overall at the complete test display because it was similar to a building representation of occlusion rather than because it was novel per se. That is, even though the nonperceivers were habituated, their ability to perceive occlusion was compromised because they failed to obtain visual information for occlusion during the time the habituation stimulus was in view. Alternatively, insufficient information acquisition during habituation might have led to a default response to the visible disjoint surfaces, which is characteristic of younger infants, yielding a novelty preference for the complete rod at test. Either scenario is consistent with the idea that efficient, targeted visual exploration may be an

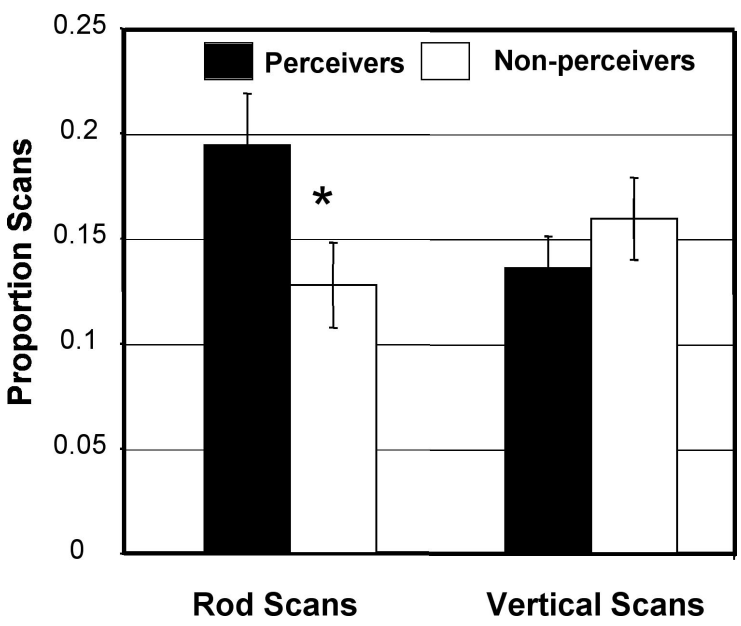

Figure 4. Average proportion of rod scans and vertical scans by each group in Experiment 1. Error bars indicate standard error of measurement. $* p<.05$. 
agent of development in perceptual completion skills. In Experiment 2, we test this hypothesis by examining differences in selection between the same groups of infants on the visual search task described previously.

\section{Experiment 2: Visual Search}

\section{Method}

Apparatus and stimuli. Each test display consisted of 27 red vertical distractor rods $\left(1.9 \times 7.0 \mathrm{~cm}\right.$ each, $\left.0.9^{\circ} \times 3.3^{\circ}\right)$ and one red target rod against a black background (see Figure 5). Distractors and targets were arranged pseudorandomly on each trial. The display was divided into 14 imaginary columns $\left(5.5 \mathrm{~cm}, 2.6^{\circ}\right.$ wide). Two rods were placed in each column in nonoverlapping positions. The target stimulus appeared in one of eight possible locations around the display center. These designated locations could also hold distractor rods, depending on the pseudorandom arrangement per trial-that is, they did not necessarily remain empty if they did not hold the target. Targets either were oriented at $30^{\circ}, 60^{\circ}$, or $90^{\circ}$ from vertical (competition condition) or were translated laterally through $6.5 \mathrm{~cm}\left(3.1^{\circ}\right)$ at speeds of $1 \mathrm{~Hz}, 1.5 \mathrm{~Hz}$, or $2 \mathrm{~Hz}$ (control condition). Each of the six possible targets $\left(30^{\circ}, 60^{\circ}, 90^{\circ}, 1 \mathrm{~Hz}, 1.5 \mathrm{~Hz}, 2 \mathrm{~Hz}\right)$ appeared in each of the eight possible locations, resulting in a total of 48 trials presented in random order for each infant.

Procedure. Two experimenters worked in concert to collect the data. The first experimenter (Scott P. Johnson) controlled the presentation of displays, and the second (Dima Amso) collected the eye movement data. Each trial was preceded by an attention-getter to draw the infant's POG to the center of the screen. When the infant fixated the attention-getter, the first experimenter pressed a key and the trial commenced. Infants viewed the display until either the first experimenter determined that a saccade was made to the target stimulus or $4 \mathrm{~s}$ had elapsed. The display was then replaced by the attention-getter to recenter the POG, and the next trial began.

Data coding. Orienting to the targets was coded frame by frame from video to determine the latency to direct the POG toward the target stimulus. Once the display appeared, the time in milliseconds to begin an eye movement toward the target stimulus was recorded. On occasion the track was lost briefly because of excessive movement or an eyeblink and was subsequently regained, followed by target acquisition. The trial ended automatically after $4 \mathrm{~s}$ if the target was not selected. Accuracy was calculated as the proportion of correct trials from the total number of trials in which data were provided $(M=69.6 \%, S D=10.2 \%)$. The probability of selecting the target by chance was $12.5 \%$ (one of eight possible target locations).

\section{Results}

Figure 6 shows mean saccade latency and proportion of targets selected as a function of trials per condition that yielded data for
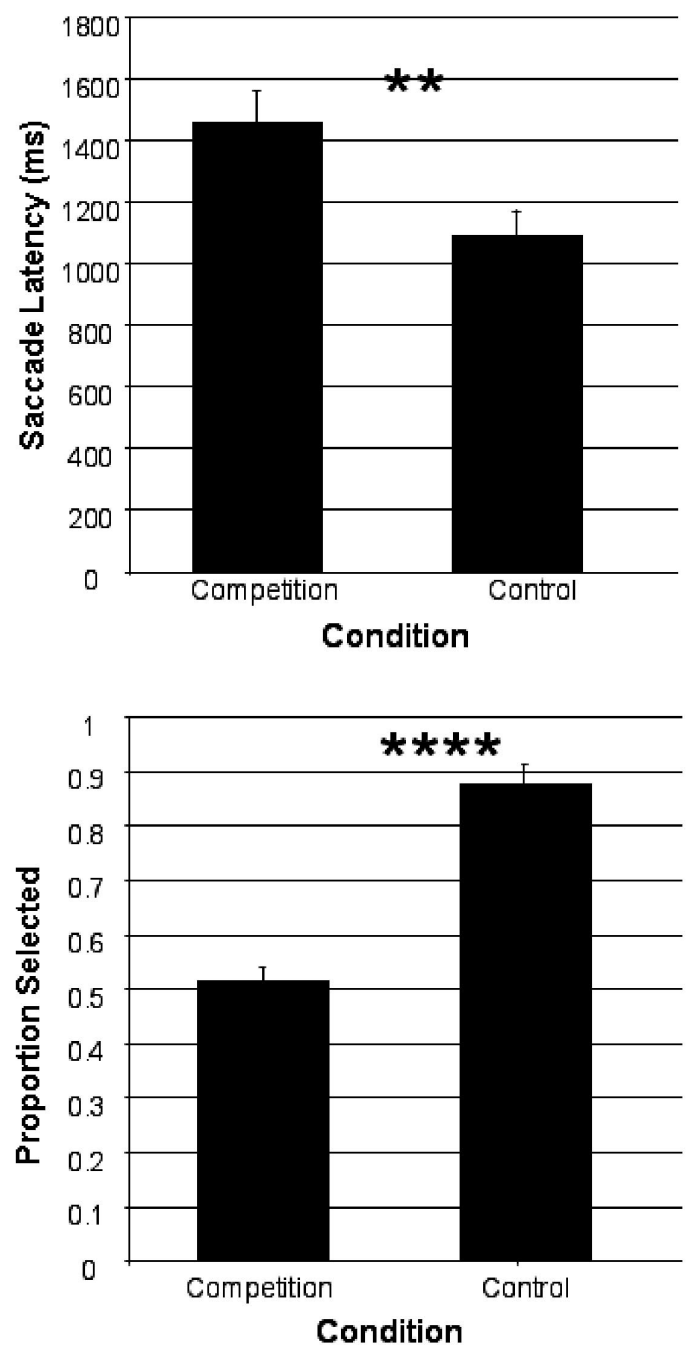

Figure 6. Top panel: Mean saccade latency performance in the competition and control conditions in Experiment 2. Lower panel: Mean proportion selected in the competition and control conditions in Experiment 2. Error bars indicate standard error of measurement. $* * p<.01 . * * * * p<$ .0001 .

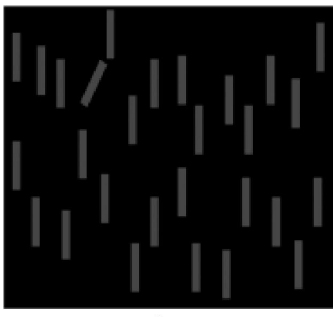

A

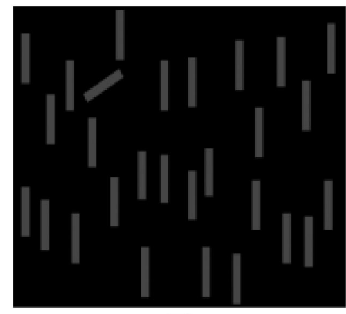

B

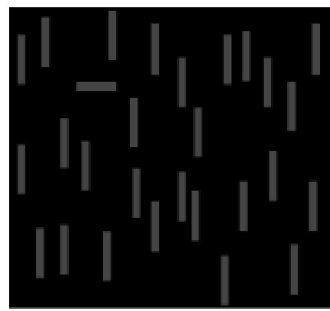

C

Figure 5. Examples of displays presented to infants in Experiment 2 in the competition task. Figure 5A: Target is oriented $30^{\circ}$ from vertical. Figure $5 \mathrm{~B}$ : Target is oriented $60^{\circ}$. Figure $5 \mathrm{C}$ : Target is oriented $90^{\circ}$. Stimuli from the control task (moving targets) are not shown. 
each infant for the competition and control conditions. Preliminary analyses incorporating sex and order of experiment (perceptual completion vs. visual search task first) revealed no significant main effects or interactions involving these variables that bore on the questions under investigation. There was no reliable difference between perceivers and nonperceivers in the number of trials that yielded data, $t(20)=0.08, n s$. Relative to the competition condition, the control condition was associated with shorter saccade latencies, $t(21)=3.67, p<.01$, and a higher proportion selected, $t(21)=8.92, p<.0001$. Taken together, these measures imply that the moving targets in the control condition were detected readily because of their salience against a static background and elicited automatic responses (Dick, Ullman, \& Sagi, 1987; McLeod, Driver, \& Crisp, 1988; Nakayama \& Silverman, 1986). The oriented targets, in contrast, might have induced relatively greater competition with the background, consistent with previous work with infants (e.g., Dannemiller, 2000; Quinn \& Bhatt, 1998) and with adults (e.g., Duncan \& Humphreys, 1989; Wolfe, 1998) showing that decreasing the difference between the target and distractors along some dimension invokes a selection cost and implies involvement of visual selective attention.

Figure 7 (top panel) shows mean saccade latency in the competition and control conditions for perceivers versus nonperceivers. Perceivers took longer to find the target in the competition condition, but performance was similar between groups in the control condition. These conclusions were confirmed with a 2 (group) $\times 3$ (orientation) mixed ANOVA on data from the competition condition, which revealed a significant main effect of group, $F(1,20)=8.06, p<.05$, as a result of longer latencies by perceivers and no other reliable effects. A 2 (group) $\times 3$ (motion: $1 \mathrm{~Hz}, 1.5 \mathrm{~Hz}$, or $2 \mathrm{~Hz}$ ) mixed ANOVA on data from the control condition revealed no reliable effects. The performance of perceivers appeared to be characterized by a speed-accuracy trade-off in the competition condition (Figure 7), and this group might have been responsible for the main effect of a latency difference between competition and control. We confirmed this conclusion by comparing search latencies in the competition versus control conditions for each group. Perceivers took longer to detect oriented targets than moving targets, $t(10)=6.06, p<.001$, but nonperceivers showed no reliable difference in latency between the conditions, $t(10)=1.17$, ns. As in Experiment 1, we found no differences between groups in the number of scans made per trial, our metric of active scanning. We computed comparisons in this measure for both competition and control conditions separately, and we also compared performance between groups in trials in which the target was selected and those in which it was not. In all these comparisons, there were no reliable differences between perceivers and nonperceivers $(t \mathrm{~s}<1.1, n s)$. These results suggest that perceivers were not simply more active in their exploration of the display but rather were slowed by their sensitivity to the competing elements.

Figure 7 (lower panel) shows the mean proportion selected in the competition and control conditions for perceivers versus nonperceivers. Performance was well above chance $(12.5 \%)$ in all four conditions: perceivers competition, $t(10)=374.08, p<.001$; perceivers control, $t(10)=464.25, p<.001$; nonperceivers competition, $t(10)=348.61, p<.001$; nonperceivers control, $t(10)=$ $184.77, p<.001$. Thus, both perceivers and nonperceivers were capable of locating the target with better than chance accuracy.
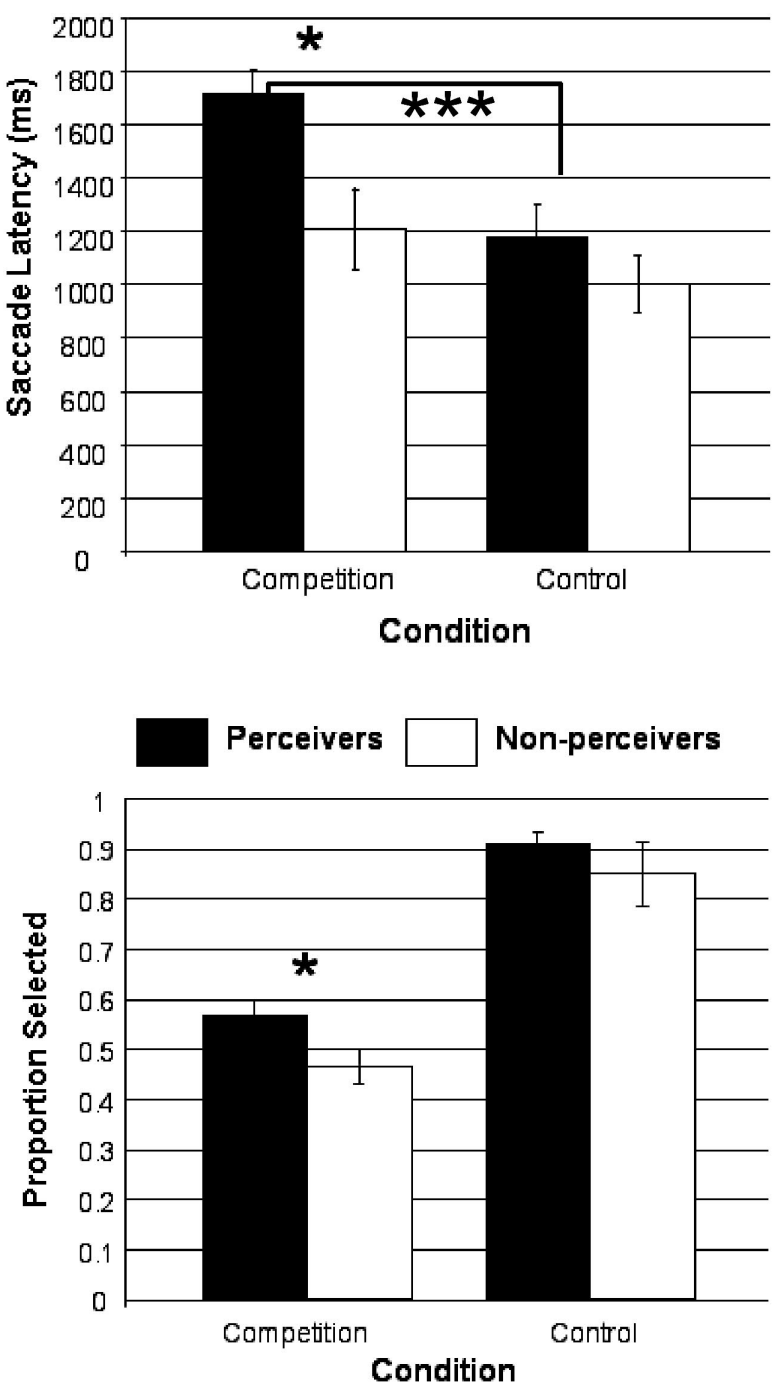

Figure 7. Top panel: Mean saccade latency performance by each group in the competition and control conditions in Experiment 2. Lower panel: Mean proportion selected by each group in the competition and control conditions in Experiment 2. Error bars indicate standard error of measurement. $* p<.05 . * * * p<.001$.

However, perceivers detected the oriented targets more frequently relative to nonperceivers, but performance was again similar between groups in the control condition. These conclusions were confirmed with a 2 (group: preference for broken vs. preference for complete) $\times 3$ (orientation: $30^{\circ}, 60^{\circ}$, or $90^{\circ}$ target) mixed ANOVA on data from the competition condition, which yielded a significant main effect of group, $F(1,20)=4.42, p<.05$, as a result of a higher number of targets detected by the perceivers, and no other reliable effects. In contrast, a 2 (group) $\times 3$ (motion: 1 $\mathrm{Hz}, 1.5 \mathrm{~Hz}$, or $2 \mathrm{~Hz}$ ) mixed ANOVA on data from the control condition revealed no reliable effects.

\section{Discussion}

As predicted, the competition condition yielded differences in performance between perceivers and nonperceivers, but no signif- 
icant differences were obtained between the groups in the control condition. Across groups, the oriented targets were selected more frequently and with more effort (as indexed by search latency) than the moving targets. As task difficulty increased, therefore, perceivers appear to have used a visual search mechanism more effectively than did nonperceivers, which led to longer latencies for selection. This indicates sensitivity to increasing competition between elements in the display. By implication, this mechanism might have been involved in targeted attention to the salient and relevant moving rod parts in the presence of competing display elements in Experiment 1.

Adult visual search studies (e.g., Treisman \& Gormican, 1988) have shown that searches for an oriented target among vertical distractors are effortless. Infants are known to be sensitive to orientation as early as 6 weeks (Braddick, Wattam-Bell, \& Atkinson, 1986), but experiments that investigate search for oriented singletons or patches of oriented bars among distractors have yielded inconsistent results. For example, in a visual search task, Rieth and Sireteanu (1994a) reported that a reliable visual preference for a display of $135^{\circ}$ elements with a single $45^{\circ}$ element emerged in middle childhood. In a familiarization-novelty preference procedure, in contrast, 5- and 6-month-olds (but not younger infants) preferred an array with a center patch of $45^{\circ}$ elements embedded among $135^{\circ}$ elements over a familiar homogeneous array of $135^{\circ}$ elements (Rieth \& Sireteanu, 1994b). Quinn and Bhatt (1998) also used a familiarization-novelty preference procedure and found that 3- and 4-month-old infants preferred a display with a single discrepant target among an array of distractors after familiarization with the homogeneous array, relative to the familiar stimulus. The reasons for these discrepancies are unclear and are beyond the scope of the present article, but it is important to point out that results of experiments that use duration of looking methods can be used to conclude only that discrimination of targets either took place or failed to obtain. It seems likely that successful discrimination in the familiarization-novelty preference procedure, nevertheless, was accomplished by effortful search.

A second important issue is that the adult (e.g., Duncan \& Humphreys, 1989) and infant (Dannemiller, 1998, 2000) visual selective attention literatures both show that alterations in the level of target-distractor similarity result in differences in search efficiency. Increasing the salience of the target relative to the background results in increasing ease of target detection. Following this logic, we manipulated target orientation offset and speeds of motion parametrically relative to the static vertical bars in the background to assess search efficiency and found no differences in accuracy or latency of search as a result of these manipulations.

It is possible that the difference in the relative salience of targets to distractors was too far above some threshold to affect search difficulty. That is not to say that infants failed to detect the differences in target orientations or motions; rather, the targetdistractor relation was not sufficiently different across varying degrees in any one condition. We chose motion as the critical feature of the control condition because a wealth of literature shows that motion is very salient on a static background to both infants and adults (Dannemiller, 2000; Dick et al., 1987; McLeod et al., 1988). Moving targets likely popped out to both perceivers and nonperceivers at a level that was perhaps close to ceiling, suggesting that even the slowest moving target was salient enough to result in pop-out. It is possible as well that detection of targets that varied in orientation was at an equivalent level of difficulty, perhaps because display elements were categorized simply as vertical or not vertical. It has been argued that two broadly tuned, orientation-sensitive channels, one near vertical and one near horizontal, can account for adult performance on simple orientation detection tasks (Foster \& Ward, 1991; Foster \& Westland, 1995). Wolfe and Friedman-Hill (1992) argued for multiple channels in adults that correspond to categorical terms such as steep, shallow, left, and right. These results cannot be applied directly to infants because of differences in orientation-based searches between groups, but it is possible that a similar kind of categorization of oriented stimuli characterized performance in the 3-month-olds we observed.

Taken together, these patterns of data suggest that differences between groups in the competition condition were not simply due to general developmental differences, such as eye movement speed, but rather were specific to the salience of the target and differences in efficiency of visual selection mechanisms. These findings begin to provide hints about an early-emerging search strategy that stems from the development of endogenous control over eye movements.

\section{General Discussion}

We investigated the hypothesis that developments in object perception skills are related to changes in visual search strategies. We found that a group of 3-month-old infants who provided evidence of perceiving the unity of disjoint surfaces also provided evidence of efficient visual selective attention in a search task. These infants, relative to infants who provided no evidence of unity perception, selected orientation-defined targets reliably more often and had longer saccade latencies. An increase in response latency with increasing task difficulty is a persistent finding in the adult visual search literature and suggests an involvement of visual selective attention (Duncan \& Humphreys, 1989; Treisman \& Gelade, 1980). Search performance did not differ between groups in the control condition (motion-defined targets), which was designed to induce a reflexive response to very salient stimuli.

Selective attention allows for the detection and subsequent processing of certain parts of a visual scene while others are ignored or suppressed. We propose that a system limited to responding to salient stimuli that pop out in the visual environment may not allow for prolonged targeting of any particular display element, impairing information extraction. In the case of perceptual completion, it may be that developments in oculomotor control, alongside attentional engagement, are related to superior information acquisition during the habituation phase of the object unity experiment. The synchronous common motion of the rod parts has been shown to be an important cue for young infants' unity perception (Jusczyk, Johnson, Spelke, \& Kennedy, 1999; Kellman \& Spelke, 1983). The ability to attend to the rod parts and ignore irrelevant yet salient display elements increases the likelihood of gathering the relevant information for the adultlike percept of unity online.

The search performance differences between the groups are consistent with biological models of oculomotor development known to take place around 3 months of age (M. H. Johnson, 1990, 2005). Control of saccades is initially exogenously driven and is 
thought to rely largely on subcortical circuitry. Reflexive eye movements involve a pathway consisting of connections from retinal ganglion cells to the lateral geniculate nucleus and the superior colliculus, with only limited input from primary cortical visual areas (Schiller, 1985, 1998). Maturation of other visual pathways supports more endogenous oculomotor control. Three cortical regions are important in attentional shifts: the frontal eye fields for the endogenous control of eye movements, the parietal cortex for covert shifts of attention, and the prefrontal cortex for endogenous control involving delays (M. H. Johnson, 2005). Endogenous control over saccadic eye movements, such as those involved in visual inspection, involves connections among visual areas V1, V2, and V4; the parietal cortex; and the frontal eye fields (Schiller, 1985, 1998). The exact timing of the development of the cortically mediated pathways remains unclear, but many aspects of oculomotor control show dramatic improvement between birth and 4 months (M. H. Johnson, 2005). One implication of these developments in oculomotor function is that the differences between groups of 3-month-olds on visual search performance may be related to different stages in the development of visual pathways underlying oculomotor control. Research on behavioral changes associated with oculomotor development has relied on effects obtained from visual selection tasks adapted from the adult attention literature. The goal of the present experiments was to elucidate a possible mechanism by which developments in visual pathways may be directly associated with more complex skills, such as object perception.

The focus of the present experiments is not on particular cognitive achievements that are expressed at a particular age but instead is on the fundamental developmental processes and mechanisms that interact to support infants' increasing facility to perceive the world in an adultlike fashion. We suggest that developments in oculomotor control that contribute to the functionality of visual selective attention skills may be related to online information-gathering behavior, which, in turn, is related to adultlike perceptual completion. However, we emphasize that infants' learning is complex, involving an interplay between emergent skills and prior information. The fact that all infants did not perceive unity via targeted visual exploration is consistent with findings in the locomotor domain showing that although locomotor experience is an agent of developmental change, it might not be necessary or sufficient for bringing change about (Campos et al., 2000).

Future work might benefit from a less categorical approach to object perception than that taken in this study. One way to accomplish this goal would be to examine how individual differences in posthabituation preference interact with oculomotor development in a longitudinal sample. Recent studies that examined the contributions of experience to cognitive development have shown that even limited exposure to task-specific information results in improved object perception skills (e.g., S. P. Johnson, Amso, \& Slemmer, 2003; Needham, 2000) and interpretation of goaldirected behavior in others (Sommerville, Woodward, \& Needham, 2005). Presumably, some of the infants we observed had enough environmental exposure to occlusion events to have an a priori understanding of object unity and hence did not need to scan the rod parts as extensively to determine their connectedness. Conversely, not all infants who scanned the rod parts efficiently in Experiment 1 provided evidence of unity perception. This, too, is consistent with the locomotor literature: In early phases of skill acquisition, prolonged exploration does not necessarily result in efficient use of available information (Adolph, 1997, 2000; Campos, Bertenthal, \& Kermoian, 1992).

Attention is critical to people's experience of the visual environment (Kanwisher \& Wojciulik, 2000). Efficient information acquisition requires visual selection mechanisms. With the emergence of selective attention, infants become active participants in their own perceptual development rather than passive recipients of information. We have shown that object information is acquired in real time and suggest that the efficiency of visual selection mechanisms can enable or restrict extraction of information from the environment for subsequent perception, encoding, and behavior.

\section{References}

Adler, S. A., Gerhardstein, P., \& Rovee-Collier, C. (1998). Levels-ofprocessing effects in infant memory? Child Development, 69, 280-294.

Adler, S. A., Inslicht, S., Rovee-Collier, C., \& Gerhardstein, P. C. (1998). Perceptual asymmetry and memory retrieval in 3-month-old infants. Infant Behavior \& Development, 21, 253-272.

Adolph, K. E. (1997). Learning in the development of infant locomotion. Monographs of the Society for Child Development, 62(3, Serial No. 251).

Adolph, K. E. (2000). Specificity of learning: Why infants fall over a veritable cliff. Psychological Science, 11, 290-295.

Adolph, K. E., Eppler, M. A., Marin, L., Weise, I. B., \& Clearfield, M. W. (2000). Exploration in the service of prospective control. Infant Behavior \& Development, 23, 441-460.

Amso, D., \& Johnson, S. P. (2005). Selection and inhibition in infancy: Evidence from the spatial negative priming paradigm. Cognition, 95, B27-B36.

Atkinson, J., Hood, B., Wattam-Bell, J., \& Braddick, O. (1992). Changes in infants' ability to switch visual attention in the first three months of life. Perception, 21, 643-653.

Bhatt, R. S., Rovee-Collier, C., \& Weiner, S. (1994). Developmental changes in the interface between perception and memory retrieval. Developmental Psychology, 30, 151-162.

Bornstein, M. H. (1985). Habituation of attention as a measure of visual information processing in human infants: Summary, systemization, and synthesis. In G. Gottlieb \& N. A. Krasnegor (Eds.), Measurement of audition and vision in the first year of postnatal life: A methodological overview (pp. 253-300). Norwood, NJ: Ablex.

Braddick, O., Wattam-Bell, J., \& Atkinson, J. (1986, April 17) Orientation-specific cortical responses develop early in life. Nature, 320, 617-619.

Bronson, G. W. (1994). Infants' transitions toward adult-like scanning. Child Development, 65, 1243-1261.

Bushnell, E. W., \& Boudreau, J. P. (1993). Motor development and the mind: The potential role of motor abilities as a determinant of aspects of perceptual development. Child Development, 64, 1005-10021.

Campos, J. J., Anderson, D. I., Barbu-Roth, M. A., Hubbard, E. M., Herternstein, M. J., \& Witherington, D. (2000). Travel broadens the mind. Infancy, 1, 149-219.

Campos, J. J., Bertenthal, B. I., \& Kermoian, R. (1992). Early experience and emotional development: The emergence of wariness of heights. Psychological Science, 3, 61-64.

Colombo, J., Ryther, J. S., Frick, J. E., \& Gifford, J. J. (1995). Visual pop-out in infants: Evidence for preattentive search in 3- and 4-month olds. Psychonomic Bulletin \& Review, 2, 266-268.

Craik, F. I. M., Govoni, R., Naveh-Benjamin, M., \& Anderson, N. D. (1996). The effects of divided attention on encoding and retrieval processes in human memory. Journal of Experimental Psychology: General, $125,159-180$. 
Dannemiller, J. L. (1998). A competition model of exogenous orienting in 3.5-month-old infants. Journal of Experimental Child Psychology, 68, $169-201$.

Dannemiller, J. L. (2000). Competition in early exogenous orienting between 7 and 21 weeks. Journal of Experimental Child Psychology, 76, 253-274.

Dannemiller, J. L. (2002). Relative color contrast drives competition in early exogenous orienting. Infancy, 3, 275-301.

Dick, M., Ullman, S., \& Sagi, D. (1987, July 24). Parallel and serial processes in motion detection. Science, 237, 400-402.

Duncan, J., \& Humphreys, G. W. (1989). Visual search and stimulus similarity. Psychological Review, 96, 433-458.

Foster, D. H., \& Ward, P. A. (1991). Horizontal-vertical filters in early vision predict anomalous line-orientation frequencies. Proceedings of the Royal Society of London, Series B: Biological Sciences, 243, 83-86.

Foster, D. H., \& Westland, S. (1995). Orientation contrast vs. orientation in line-target detection. Vision Research, 35, 733-738.

Gibson, E. J. (1988). Exploratory behavior in the development of perceiving, acting, and acquiring of knowledge. Annual Review of Psychology, $39,1-41$.

Gibson, E. J., \& Pick, A. D. (2000). An ecological approach to perceptual learning and development. New York: Oxford University Press.

Johnson, M. H. (1990). Cortical maturation and the development of visual attention in infancy. Journal of Cognitive Neuroscience, 2, 81-95.

Johnson, M. H. (2005). Developmental cognitive neuroscience (2nd ed.). London: Blackwell.

Johnson, M. H., \& Tucker, L. A. (1996). The developmental and temporal dynamics of spatial orienting in infants. Journal of Experimental Child Psychology, 63, 171-188.

Johnson, S. P. (1997). Young infants' perception of object unity: Implications for development of attentional and cognitive skills. Current Directions in Psychological Science, 6, 5-11.

Johnson, S. P. (2000). The development of visual surface perception: Insights into the ontogeny of knowledge. In C. Rovee-Collier, L. Lipsitt, $\&$ H. Hayne (Eds.), Progress in infancy research (Vol. 1, pp. 113-154). Mahwah, NJ: Erlbaum.

Johnson, S. P. (2004). Development of perceptual completion in infancy. Psychological Science, 15, 769-775.

Johnson, S. P., Amso, D., \& Slemmer, J. A. (2003). Development of object concepts in infancy: Evidence for early learning in an eye tracking paradigm. Proceedings of the National Academy of Sciences USA, 100, 10568-10573.

Johnson, S. P., \& Aslin, R. N. (1995). Perception of object unity in 2-month-old infants. Developmental Psychology, 31, 739-745.

Johnson, S. P., Slemmer, J. A., \& Amso, D. (2004). Where infants look determines how they see: Eye movements and object perception performance in 3-month-olds. Infancy, 6, 185-201.

Jusczyk, P. W., Johnson, S. P., Spelke, E. S., \& Kennedy, L. J. (1999). Synchronous change and perception of object unity: Evidence from adults and infants. Cognition, 71, 257-288.

Kanwisher, N., \& Wojciulik, E. (2000). Visual attention: Insights from brain imaging. Nature Reviews Neuroscience, 1, 91-100.

Kellman, P. J., \& Spelke, E. S. (1983). Perception of partly occluded objects in infancy. Cognitive Psychology, 15, 483-524.

McLeod, P., Driver, J., \& Crisp, J. (1988, March 10). Visual search for conjunctions of movement and form is parallel. Nature, 332, 154-155.

Nakayama, K., \& Silverman, G. H. (1986, March 20). Serial and parallel processing of visual feature conjunctions. Nature, 320, 264-265.

Needham, A. (2000). Improvements in visual exploration skills may facilitate the development of object segregation in early infancy. Journal of Cognition and Development, 1, 131-156.

Needham, A., Barrett, T., \& Peterman, K. (2002). A pick-me-up for infants' exploratory skills: Early simulated experiences reaching for objects using "sticky mittens" enhances young infants' object exploration skills. Infant Behavior \& Development, 25, 279-295.

Neisser, R. (1967). Cognitive psychology. New York: Appleton-CenturyCrofts.

Piaget, J. (1954). The construction of reality in the child (M. Cook, Trans.). New York: Basic Books. (Original work published 1937)

Quinn, P. C., \& Bhatt, R. S. (1998). Visual pop-out in young infants: Convergent evidence and an extension. Infant Behavior \& Development, 21, 273-288.

Rieth, C., \& Sireteanu, R. (1994a). Texture segmentation and "pop-out" in infants and children: The effect of test field size. Spatial Vision, 8, 173-191.

Rieth, C., \& Sireteanu, R. (1994b). Texture segmentation and visual search based on orientation contrast: An infant study with the familiarizationnovelty preference method. Infant Behavior \& Development, 17, 359-369.

Ross, S., \& Dannemiller, J. L. (1999). Color contrast, luminance contrast, and competition within exogenous orienting in 3.5-month-old infants. Infant Behavior \& Development, 22, 383-404.

Rovee-Collier, C., Bhatt, R. S., \& Chazin, C. (1996). Set size, novelty, and visual pop-out in infancy. Journal of Experimental Psychology: Human Perception and Performance, 22, 1178-1187.

Rovee-Collier, C., Hankins, E. M., \& Bhatt, R. S. (1992). Textons, visual pop-out effects and object recognition in infancy. Journal of Experimental Psychology: General, 121, 436-446.

Schiller, P. H. (1985). A model for the generation of visually guided saccadic eye movements. In D. Rose \& V. G. Dobson (Eds.), Models of the visual cortex (pp. 62-70). New York: Wiley.

Schiller, P. H. (1998). The neural control of visually guided eye movements. In J. E. Richards (Ed.), Cognitive neuroscience of attention: A developmental perspective (pp. 3-50). Mahwah, NJ: Erlbaum.

Slater, A. (1995). Visual perception and memory at birth. In C. RoveeCollier \& L. P. Lipsitt (Eds.), Advances in infancy research (Vol. 9, pp. 107-162). Norwood, NJ: Ablex.

Slater, A., Johnson, S. P., Brown, E., \& Badenoch, M. (1996). Newborn infants' perception of partly occluded objects. Infant Behavior \& Development, 19, 145-148.

Slater, A., Morison, V., Somers, M., Mattock, A., Brown, E., \& Taylor, D. (1990). Newborn and older infants' perception of partly occluded objects. Infant Behavior \& Development, 13, 33-49.

Sokolov, E. (1963). Perception and the conditioned reflex. Oxford, England: Pergamon.

Sommerville, J. A., Woodward, A. L., \& Needham, A. (2005). Action experience alters 3-month-old infants' perception of others actions. Cognition, 96, B1-B11.

Treisman, A. (1988). Features and objects: The fourteenth Bartlett memorial lecture. Quarterly Journal of Experimental Psychology: Human Experimental Psychology, 40(A), 210-237.

Treisman, A. (1992). Perceiving and re-perceiving objects. American Psychologist, 47, 862-875.

Treisman, A., \& Gelade, G. (1980). A feature integration theory of attention. Cognitive Psychology, 12, 97-136.

Treisman, A., \& Gormican, S. (1988). Feature analysis in early vision: Evidence from search asymmetries. Psychological Review, 95, 15-48.

Wolfe, J. M. (1998). Visual search. In H. Pashler (Ed.), Attention (pp. 13-74). Hove, East Sussex, England: Psychology Press.

Wolfe, J. M., \& Friedman-Hill, S. R. (1992). Visual search for orientation: The role of angular relations between targets and distracters. Spatial Vision, 6, 199-208.

Zenger, B., Braun, J., \& Koch, C. (2000). Attentional effects on contrast detection in the presence of surround masks. Vision Research, 40, $3717-3724$.

Received June 22, 2005

Revision received May 8, 2006

Accepted May 12, 2006 